\title{
Valores energéticos de diferentes alimentos de origem animal para aves ${ }^{1}$
}

\section{Ricardo Vianna Nunes ${ }^{2}$, Horacio Santiago Rostagno ${ }^{3}$, Paulo Cezar Gomes ${ }^{3}$, Christiane Garcia Vilela Nunes ${ }^{4}$, Luiz Fernando Teixeira Albino ${ }^{3}$, Paulo Cesar Pozza², Marli Arena Dionizio 4 , Marcelle Santana de Araújo 4}

\author{
1 Parte da tese apresentada pelo primeiro autor à UFV para obtenção do título de Doctor Scientiae. \\ 2 Centro Ciências Agrárias, UNIOESTE - 85960-000 - Marechal Cândido Rondon, PR. \\ ${ }^{3}$ Departamento de Zootecnia, UFV - 36571-000 - Viçosa, MG. \\ ${ }^{4}$ Pós-Graduação em Zootecnia, UFV - 36571-000 - Viçosa, MG.
}

RESUMO - Objetivou-se determinar, utilizando-se o método tradicional de coleta total, os valores de energia metabolizável aparente (EMA), aparente corrigida $\left(\mathrm{EMA}_{\mathrm{n}}\right)$, verdadeira (EMV) e verdadeira corrigida $\left(\mathrm{EMV}_{\mathrm{n}}\right)$, bem como os coeficientes de metabolizabilidade da EB, de 11 alimentos. Avaliaram-se amostras de três farinhas de vísceras de aves (FVA), três farinhas de penas (FP), duas farinhas de carne e ossos (FCO), duas farinhas de vísceras suínas (FVS) e uma farinha de penas e vísceras (FPV) de diferentes procedências. Foram utilizados 360 frangos de corte machos Ross com 21 dias de idade, distribuídos em blocos ao acaso em 11 tratamentos e uma ração-referência, com duas repetições por bloco e cinco aves por unidade experimental. Cada alimento substituiu em $20 \%$ a ração-referência. As aves receberam ração à vontade por 12 dias, sendo os cinco dias finais destinados à coleta total de excretas. Durante os cinco dias de coleta, 30 aves (distribuídas em seis gaiolas) foram mantidas em jejum por 72 horas, sendo as 24 horas iniciais para esvaziamento do trato gastrointestinal e as 48 horas restantes para coleta das excretas, que foram quantificadas e extrapoladas para cinco dias. Os valores de EMA e EMA expressos em kcal/kg de MS, foram em média de 1.647 e 1.422 para a FCO, de 2.143 e 1.875 para a FP, de 3.062 e 2.844 para a FVA, de 1.889 e 1.779 para a FVS e de 2.497 a 2.209 para a FPV. Os valores de EMV e EMV , expressos em $\mathrm{kcal} / \mathrm{kg}$ de MS, foram em média de 1.753 e 1.462 para a FCO, de 2.269 e 1.452 para a FP, de 3.211 e 2.740 para a FVA, de 2.002 e 1.562 para a FVS e de 2.615 e 1.944 para a FPV.

Palavras-chave: energia metabolizável aparente, energia metabolizável verdadeira, coeficientes de metabolizabilidade, frangos de corte

\section{Energetic values of different feedstuffs of animal origin for poultry}

ABSTRACT - The traditional method of total collection was used to determine the values of apparent metabolizable energy $(\mathrm{AME})$, corrected apparent energy $\left(\mathrm{AME}_{\mathrm{n}}\right)$, true metabolizable energy (TME) and corrected true energy $\left(\mathrm{TME}_{\mathrm{n}}\right)$ of eleven feedstuffs and the respective coefficients of metabolizability of GE. The feedstuffs were as follows: three samples of poultry by-product (PBP), three of feather meal (FM), two of meat and bone meal (MBM), two of swine viscera meal (SVM) and one of viscera and feather meal (VFM) of different origins. Three hundred and sixty male Ross broiler chickens, with 21 days old, were assigned to a randomized block design with eleven treatments and one control, with two replicates per block and five birds per experimental unit. The feedstuffs replaced $20 \%$ of the control. The birds were fed ad libitum during 12 days (last five days for total feces collection). During the collection, 30 poultry were allotted to six cages and fasted for 72 hours (the first 24 hours were for the gastrointestinal emptying and the 48 hours remaining for feces collection, that were quantified and extrapolated for five days). The average values of $\mathrm{AME}$ and $\mathrm{AME}_{\mathrm{n}}$ expressed in $\mathrm{kcal} / \mathrm{kg}$ on DM basis were as follows: 1,647 and 1,422 for MBM, 2,143 and 1,875 for FP, 3,062 and 2,844 for PBP, 1,889 and 1,779 for SVM and of 2,497 and 2,209 for VFM. The average values of TME and $\mathrm{TME}_{\mathrm{n}}$ expressed in $\mathrm{kcal} / \mathrm{kg}$ of $\mathrm{DM}$, were: 1,753 and 1,462 for MBM, 2,269 and 1,452 for FM, 3,211 and 2,740 for PBM, 2,002 and 1,562 for SVM and 2,615 and 1,944 for VFM.

Key Words: apparent metabolizable energy, broiler chicks, coefficients of metabolizability, true metabolizable energy

\section{Introdução}

A energia é um componente fundamental na elaboração de rações avícolas e não é um nutriente, mas o resultado da oxidação dos nutrientes durante o metabolismo animal
(NRC, 1994). Os animais ingerem alimentos para suprir suas necessidades energéticas e, portanto, é necessário manter a relação entre a energia disponível e os nutrientes que compõem a ração. Além disso, tanto a energia metabolizável verdadeira (EMV) quanto a aparente (EMA) devem ser 
corrigidas pelo balanço de nitrogênio para melhor representar a energia efetivamente utilizada pela ave.

O conhecimento da composição química e do conteúdo energético dos alimentos é de grande importância para a formulação de rações. A composição dos subprodutos de origem animal pode variar conforme o processamento a que são submetidos e ao tipo e à proporção de seus componentes originais.

As farinhas de origem animal são ingredientes importantes quanto aos aspectos econômico, sanitário e nutricional. Além disso, seu uso na formulação de dietas é facilitado porque esses alimentos contêm aminoácidos, energia, cálcio e fósforo em quantidades apreciáveis. Entretanto, sua utilização nutricional depende principalmente do conhecimento de suas composições química e energética.

Considerando o teor poluente dos subprodutos de abatedouros, sua utilização nas rações tem papel importante na reciclagem de nutrientes e na preservação do meio ambiente.

Este trabalho foi realizado com o objetivo de determinar os valores de energia metabolizável aparente (EMA), energia metabolizável aparente corrigida pelo balanço de nitrogênio $\left(\mathrm{EMA}_{\mathrm{n}}\right)$, energia metabolizável verdadeira (EMV) e energia metabolizável verdadeira corrigida pelo balanço de nitrogênio $\left(\mathrm{EMV}_{\mathrm{n}}\right)$, bem como os coeficientes de metabolizabilidade da energia bruta (EB), de diferentes alimentos de origem animal para aves.

\section{Material e Métodos}

O ensaio biológico foi realizado no Setor de Avicultura do Departamento de Zootecnia da Universidade Federal de Viçosa - MG utilizando-se o método tradicional de coleta total de excretas. As temperaturas média, máxima e mínima e a umidade relativa média do ar no interior da sala utilizada para os estudos de metabolismo, durante a fase experimental, foram de $29,0^{\circ} \mathrm{C} ; 22,7^{\circ} \mathrm{C}$ e $57,9 \%$, respectivamente.

Foram avaliadas amostras de três farinhas de penas (FP 1, 2 e 3), três farinhas de vísceras de aves (FVA 1, 2 e 3), duas farinhas de vísceras suínas (FVS 1 e 2), duas farinhas de carne e ossos (FCO 1 e 2) e uma farinha de penas e vísceras (FPV) provenientes de diferentes fornecedores. Determinaram-se os valores de MS, N, EE, FB, FDN, FDA, cinzas, Ca, $\mathrm{P}$ e $\mathrm{Mg}$, segundo as técnicas descritas por Silva \& Queiroz (2002). Também foi determinado o diâmetro geométrico médio das partículas dos alimentos (DGM) utilizando-se a técnica adaptada de Zanotto \& Bellaver (1996).

Para determinação dos valores de EM, foram utilizados 360 frangos de corte machos da linhagem Ross, com 21 dias de idade e peso médio de $586 \mathrm{~g}$. Os tratamentos consistiram dos alimentos, que substituíram em $20 \%$ uma ração-referência (Tabela 1) formulada segundo exigências descritas por Rostagno et al. (2000), exceto para PB.

O delineamento experimental foi em blocos ao acaso, com duas repetições por bloco, 12 rações e cinco aves por unidade experimental. As aves receberam ração inicial para frangos de corte até o $21^{\circ}$ dia de idade, quando foram transferidas para baterias de estrutura metálica. As aves foram mantidas em sistema de iluminação contínua (24 horas de luz) por todo o período experimental e, durante 12 dias (sete de adaptação e cinco de coleta total de excretas), receberam água e ração à vontade. As coletas foram realizadas duas vezes ao dia, às 8 e 17 h. Ao término do experimento, foi determinada a quantidade de ração consumida por unidade experimental durante os cinco dias de coleta.

Para determinação das perdas fecais endógenas e urinárias metabólicas, 30 aves de mesma idade, sexo e linhagem foram mantidas em jejum durante 72 horas ( 24 para esvaziamento do trato gastrointestinal e 48 para coleta total de excretas). Após o término da última coleta, as excretas foram pesadas e os valores obtidos foram extrapolados para uma produção de cinco dias.

As excretas coletadas em toda a fase experimental foram acondicionadas em sacos plásticos devidamente identificados e armazenadas em freezer até o final do período de coleta. Posteriormente, foram descongeladas, pesadas e homogeneizadas, sendo retiradas amostras para análises laboratoriais. Efetuou-se a pré-secagem em estufas ventiladas a $55^{\circ} \mathrm{C}$, com subseqüente análise dos teores de MS a $105^{\circ} \mathrm{C}$, de nitrogênio e EB.

Uma vez obtidos os resultados das análises laboratoriais dos alimentos, da ração-referência e das excretas, foram calculados os valores de EMA, EMA , EMV e $\mathrm{EMV}_{\mathrm{n}}$ utilizando-se equações propostas por Matterson et al. (1965).

Os coeficientes de metabolizabilidade da EB, em relação aos valores de EMA, EMA ${ }_{n}, E$ V e EMV ${ }_{n}$, foram calculados segundo Leeson \& Summers (2001). O coeficiente de metabolizabilidade indica a porcentagem da EB metabolizada em forma de EMA, EMA ${ }_{n}, E_{V}$ e EMV , calculada utilizando-se os valores de EM divididos pela EB referente a cada alimento.

Com base nos valores de EB e de EM dos alimentos, foram calculados os coeficientes de metabolizabilidade dos valores energéticos. Como procedimento estatístico, aplicou-se o teste de comparação de médias Student Newman-Keuls, a 5\% de probabilidade, entre os valores médios dos coeficientes de metabolizabilidade, por meio 
Tabela 1 - Composição da ração-referência, em porcentagem da matéria natural

Table 1 - Ingredient composition of the reference diet, \% as-fed basis

\begin{tabular}{lc}
\hline Ingrediente & $(\%)$ \\
Ingredient & \\
\hline Milho (Corn) & 69,707 \\
Farelo de soja (Soybean meal) & 24,220 \\
Óleo vegetal (Vegetable oil) & 1,500 \\
Fosfato bicálcico (Dicalcium phosphate) & 1,643 \\
Calcário (Limestone) & 1,020 \\
Sal (Salt) & 0,390 \\
Suplemento mineral ${ }^{1}$ (Mineral mix) & 0,060 \\
Suplemento vitamínico (Vitamin mix) & 0,130 \\
DL-metionina (99\%) (DL-methionine) & 0,255 \\
L-lisina.HCl (98\%) (L-lysine) & 0,298 \\
L-treonina (99\%) (L-threonine) & 0,040 \\
Cloreto de colina (60\%) (Choline chloride) & 0,060 \\
Anticoccidiano ${ }^{3}$ (Anticoccidial) & 0,055 \\
Promotor de crescimento ${ }^{4}$ (Growth promoter) & 0,002 \\
Antioxidante ${ }^{5}$ (Antioxidant) & 0,020 \\
Óxido de cromo (Cr ${ }_{2} \mathrm{O}_{3}$ ) & 0,600 \\
Total & 100,00 \\
\hline
\end{tabular}

Valores calculados de energia e nutrientes

Calculated values of energy and nutrients

\begin{tabular}{ll}
\hline Proteína bruta (\%) (Crude protein) & 17,05 \\
Energia metabolizável (kcal/kg) (Metabolizable energy) & 3.055 \\
Metionina digestível (\%) (Digestible methionine) & 0,505 \\
Met. + Cis. digestível (\%) (Digestible meth. + cys.) & 0,750 \\
Lisina digestível (\%) (Digestible lysine) & 1,046 \\
Treonina digestível (\%) (Digestible threonine) & 0,608 \\
Triptofano digestível (\%) (Digestible tryptophan) & 0,179 \\
Cálcio (\%) (Calcium) & 0,898 \\
Fósforo disponível (\%) (Avaliable phosphorus) & 0,406 \\
Sódio (\%) (Sodium) & 0,192 \\
Potássio (\%) (Potassium) & 0,655
\end{tabular}

${ }^{1}$ Conteúdo/kg (Content/kg): Fe - 100 g; Co - 2,0 g; Cu - 20 g; Mn - 160 g; $\mathrm{Zn}-100 \mathrm{~g} ; \mathrm{I}$ - 2g; excip. q.s.p. - $500 \mathrm{~g}$.

2 Conteúdo/kg (Content $/ \mathrm{kg}$ ): vit. A - 10.000.000 UI; vit. $\mathrm{D}_{3}-2.000 .000 \mathrm{UI}$; vit. E - 30.000 Ul; vit. $B_{1}-2$ g; vit. $B_{2}-6$ g; vit. $B_{6}-4$ g; vit. $B_{12}-0,015 \mathrm{~g}$;

ác. pantotênico (pantothenic acid) - $12 \mathrm{~g}$; vit. $\mathrm{K}_{3}-3 \mathrm{~g}$; ác. fólico (folic acid) -

$1 \mathrm{~g}$; ác. nicotínico (nicotinic acid) - $50 \mathrm{~g}$; Se - 0,25 g; excip. q.s.p. $-1.000 \mathrm{~g}$.

${ }^{3}$ Coxistac.

${ }^{4}$ Virginiamicina $2 \%$ (virginiamicin)

${ }^{5}$ Butil Hidroxi Tolueno (BHT).

do Sistema de Análises Estatísticas e Genéticas - SAEG (UFV, 1999).

\section{Resultados e Discussão}

A composição química variou em um mesmo alimento (Tabela 2), observando-se que a FPV e as FP apresentaram os maiores valores de $\mathrm{PB}$ e as FCO, a FVS e as FVA, os maiores valores para cinzas (13,02 a 39,03\%).

Os valores de FB e cinzas das FP 1 e FP 3 e de EE da FP 1 e da FP 2 mantiveram-se dentro dos padrões estabelecidos pela ANFAR (1985), que recomenda para as FP máximo de $2,5 \%$ de EE, $1,5 \%$ de FB e $5 \%$ de cinzas. Contudo, a EMBRAPA (1991) cita que uma farinha de penas pode conter $7,27 \%$ de EE.
Quanto às FVA e as FVS, apenas as FVS apresentaram níveis protéicos abaixo dos recomendados pela ANFAR (1985). Os teores de EE e de cinzas foram muito acima das especificações e apenas os valores de FB estiveram próximos aos preconizados pela ANFAR (1985), segundo a qual uma FV deve conter no mínimo $65 \%$ de $\mathrm{PB}$ e no máximo $1 \%$ de FB e $7 \%$ de cinzas, podendo o conteúdo de EE variar de 8 a $12 \%$.

Ao comparar os resultados obtidos neste estudo com os da literatura (Rostagno et al., 2000; EMBRAPA, 1991; Teixeira, 1998; Scott et al., 1982; NRC, 1994 e 1998; Leeson \& Summers, 2001), observou-se grande variação nos valores da composição química dos alimentos estudados, havendo, inclusive, diferença para um mesmo alimento quando comparados entre si.

As diferenças na composição química dos alimentos já eram esperadas, pois as matérias-primas utilizadas na constituição das farinhas eram diferentes e não há padronização desses produtos, tendo em vista os fatores operacionais e os constituintes das farinhas. Além disso, o tipo de processamento que cada farinha recebe pode influenciar diretamente a composição química e, conseqüentemente, a qualidade desses alimentos (Albino \& Silva, 1996).

Pelo diâmetro geométrico médio das partículas (DGM), classificaram-se os alimentos FCO 1 e FVA 1 como de granulometria fina e os demais, como de granulometria média. Segundo Zanotto \& Bellaver (1996), os alimentos são classificados como de granulometria fina quando apresentam DGM é inferior a $0,60 \mathrm{~mm}$, média quando o DGM é de 0,60 a 2,00 $\mathrm{mm}$ e grossa quando o DGM é superior a 2,00 $\mathrm{mm}$.

Os valores de EMA, EMA ${ }_{n}, E M V$ e $E M V_{n}$ e seus respectivos desvios-padrão são apresentados na Tabela 3. As variações foram de 1.408 a 3.448 e de 1.195 a $3.069 \mathrm{kcal} / \mathrm{kg}$ de MS para os valores de EMA e EMA $\mathrm{n}$, respectivamente; de 1.513 a 3.605 e de 947 a $3.219 \mathrm{kcal} / \mathrm{kg}$ de MS para os valores de EMV e de $\mathrm{EMV}_{\mathrm{n}}$, respectivamente.

Os valores de EMA foram superiores em $9,58 \%$ aos de EMA $_{\mathrm{n}}$ e os de EMV em 22,55\% aos de $\mathrm{EMV}_{\mathrm{n}}$. Os valores de EMA foram normais e, quando determinados pelo método tradicional com pintos, foram superiores aos de $\mathrm{EMA}_{\mathrm{n}}$, tendo em vista a maior retenção de nitrogênio pelas aves, superioridade mais acentuada com a correção pelas perdas endógenas e metabólicas. De acordo com Coelho (1983), em níveis normais de consumo, as perdas de energia fecal metabólica (EFm) e de energia urinária endógena (EUe) são pequenas em relação à excreção de energia proveniente do alimento e têm pouca influência nos valores de EM obtidos pelo método tradicional. Dessa forma, observou-se que, ao corrigir os valores de EM pelas perdas endógenas, ocorreu aumento de aproximadamente 5,20\% nos valores de EMV em relação aos valores de EMA e redução de aproximada- 
Tabela 2 - Composição química dos alimentos e diâmetro geométrico médio das partículas (DGM), expressos na matéria natural Table 2 - Chemical composition and average geometric diameter (MGD) of feedstuffs, as-fed basis

\begin{tabular}{|c|c|c|c|c|c|c|c|c|c|}
\hline $\begin{array}{l}\text { Alimento } \\
\text { Feedstuff }\end{array}$ & $\begin{array}{l}\mathrm{MS} \% \\
\% D M\end{array}$ & $\begin{array}{l}\mathrm{PB} \% \\
\% C P\end{array}$ & $\mathrm{EE} \%$ & $\begin{array}{l}\mathrm{FB} \% \\
\% C F\end{array}$ & $\begin{array}{c}\text { MM\% } \\
\% \text { ash }\end{array}$ & $\mathrm{P} \%$ & $\mathrm{Ca} \%$ & $\mathrm{Mg} \%$ & $\begin{array}{c}\mathrm{DGM}^{1} \\
M G D\end{array}$ \\
\hline $\mathrm{FCO} 1(M B M 1)$ & 92,76 & 40,30 & 11,29 & 2,06 & 36,02 & 4,75 & 6,42 & 0,54 & 403,7 \\
\hline FCO $2(M B M 2)$ & 92,65 & 40,88 & 9,95 & 1,28 & 39,03 & 6,48 & 7,41 & 0,42 & 752,0 \\
\hline FP $1(F M 1)$ & 78,11 & 71,52 & 1,99 & 1,30 & 2,75 & 0,30 & 0,35 & 0,09 & 1269,2 \\
\hline FP $2(F M 2)$ & 91,37 & 77,44 & 2,51 & 0,87 & 7,32 & 0,43 & 0,36 & 0,09 & 1033,4 \\
\hline FP 3 (FM 3) & 90,93 & 80,58 & 3,24 & 0,80 & 2,66 & 0,36 & 0,28 & 0,10 & 676,7 \\
\hline FVA 1 ( $P B P$ 1) & 92,66 & 54,07 & 16,47 & 1,94 & 19,34 & 3,07 & 3,57 & 0,30 & 487,4 \\
\hline FVA 2 (PBP 2) & 91,56 & 49,19 & 18,44 & 1,64 & 19,39 & 2,72 & 5,35 & 0,47 & 1229,1 \\
\hline FVA 3 (PBP 3) & 91,41 & 59,54 & 13,29 & 1,53 & 13,02 & 2,20 & 2,06 & 0,25 & 1393,3 \\
\hline FVS 1 (SVM 1) & 96,54 & 42,46 & 15,63 & 1,81 & 32,73 & 3,66 & 7,04 & 0,35 & 1680,7 \\
\hline FVS 2 (SVM 2) & 95,31 & 47,60 & 13,26 & 2,71 & 31,58 & 5,94 & 6,37 & 0,45 & 1756,0 \\
\hline FPV (VFM) & 91,28 & 71,40 & 10,40 & 1,05 & 4,34 & 0,72 & 2,32 & 0,47 & 891,8 \\
\hline
\end{tabular}

${ }^{1} \mathrm{DGM}(\mathrm{mm})$.

Tabela 3 - Valores de energia metabolizável aparente $(E M A)$, aparente corrigida $\left(E_{n} A_{n}\right)$, verdadeira $(E M V)$ e verdadeira corrigida $\left(E M V_{n}\right)^{1}$ e seus respectivos desvios-padrão

Table 3 - Values of apparent metabolizable energy (AME), corrected apparent energy $\left(A M E_{n}\right)$, true energy (TME) and corrected true energy $\left(T M E_{n}\right)^{1}$ and the respective standard deviations (SD)

\begin{tabular}{|c|c|c|c|c|}
\hline $\begin{array}{l}\text { Alimento } \\
\text { Feedstuff }\end{array}$ & $\begin{array}{l}\text { EMA } \\
A M E\end{array}$ & $\begin{array}{c}\mathrm{EMA}_{\mathrm{n}} \\
A M E_{n}\end{array}$ & $\begin{array}{l}\text { EMV } \\
T M E\end{array}$ & $\begin{array}{l}\mathrm{EMV}_{\mathrm{n}} \\
T M E_{n}\end{array}$ \\
\hline $\mathrm{FCO} 1(M B M 1)$ & $1.408 \pm 161^{2}$ & $1.195 \pm 135$ & $1.513 \pm 148$ & $1.216 \pm 171$ \\
\hline FCO $2(M B M 2)$ & $1.885 \pm 308$ & $1.650 \pm 269$ & $1.992 \pm 318$ & $1.709 \pm 364$ \\
\hline $\mathrm{FP} 1(F M 1)$ & $1.772 \pm 178$ & $1.573 \pm 191$ & $1.880 \pm 192$ & $947 \pm 200$ \\
\hline $\mathrm{FP} 2$ (FM 2) & $2.187 \pm 153$ & $1.924 \pm 163$ & $2.279 \pm 165$ & $1.481 \pm 171$ \\
\hline FP 3 (FM 3) & $2.472 \pm 259$ & $2.130 \pm 222$ & $2.647 \pm 238$ & $1.929 \pm 282$ \\
\hline FVA $1(P B P 1)$ & $2.920 \pm 290$ & $2.750 \pm 279$ & $3.065 \pm 287$ & $2.554 \pm 303$ \\
\hline FVA $2(P B P 2)$ & $2.818 \pm 294$ & $2.713 \pm 283$ & $2.965 \pm 290$ & $2.448 \pm 307$ \\
\hline FVA 3 ( $P B P 3)$ & $3.448 \pm 562$ & $3.069 \pm 465$ & $3.604 \pm 580$ & $3.219 \pm 691$ \\
\hline FVS 1 (SVM 1) & $1.920 \pm 482$ & $1.844 \pm 436$ & $2.033 \pm 489$ & $1.595 \pm 550$ \\
\hline FVS 2 (SVM 2) & $1.857 \pm 488$ & $1.714 \pm 441$ & $1.971 \pm 495$ & $1.528 \pm 557$ \\
\hline $\mathrm{FPV}(V F M)$ & $2.497 \pm 568$ & $2.209 \pm 466$ & $2.615 \pm 556$ & $1.944 \pm 653$ \\
\hline
\end{tabular}

1 Valores expressos em $\mathrm{kcal} / \mathrm{kg}$ de MS (values expressed in $\mathrm{kcal} / \mathrm{kg}$ of DM).

2 Desvio-padrão (standard desviation).

mente $9,66 \%$ nos valores de $\mathrm{EMV}_{\mathrm{n}}$ em relação aos valores de $\mathrm{EMA}_{\mathrm{n}}$. Esta redução nos valores energéticos, quando corrigida pelo balanço de nitrogênio, é provocada pelo efeito positivo das aves na retenção de nitrogênio quando alimentadas à vontade.

Nascimento et al. (2002) notaram redução nos valores de $\mathrm{EMA}_{\mathrm{n}}$ das farinhas de vísceras, quando comparados aos de EMA, e encontraram para as FP valores médios de $\mathrm{EMA}_{n}$ superiores ao de EMA, demonstrando claramente a interferência do balanço de nitrogênio nos valores energéticos dos alimentos. Esses autores encontraram diferenças entre os valores de EMV, que, algumas vezes, foram superiores e outras inferiores aos de EMA. Segundo Farrel et al. (1991), os valores de EMA $_{n}$ e EMA podem ser influenciados pelo consumo de ração, que pode interferir nas perdas energéticas metabólicas e endógenas.

Nas tabelas de composição química e energética dos alimentos, são encontrados com maior freqüência os valores de $\mathrm{EMA}_{\mathrm{n}}$, pois são utilizados diretamente na formulação de rações. Os valores de $\mathrm{EMA}_{\mathrm{n}}$ para a FCO1 e FCO2 foram de 1.195 e $1.650 \mathrm{kcal} / \mathrm{kg}$ de MS, respectivamente, inferiores aos propostos por Rostagno et al. (2000) e NRC (1994), que descrevem que a FCO contém, em média, $2.200 \mathrm{kcal} / \mathrm{kg}$ de MS. Para os alimentos FVA e FVS, os valores de EMA $_{n}$ foram, em média, 3.062 e $1.889 \mathrm{kcal} / \mathrm{kg}$ de MS, respectivamente. Esses valores de FVA foram semelhantes (Nascimento et al., 2002; Rostagno et al., 2000; NRC, 1994; Leeson \& Summers, 2001), enquanto os de EMA $_{n}$ da FVS foram inferiores aos da literatura consultada.

As FP apresentaram valores de $\mathrm{EMA}_{\mathrm{n}}$ de 1.573 a $2.130 \mathrm{kcal} / \mathrm{kg}$ de MS e somente a FP 3 apresentou valor de EMA $_{n}$ próximo ao descrito pelo NRC (1994), que propõe $2.538 \mathrm{kcal} / \mathrm{kg}$ de MS.

Considerando a média dos valores de $\mathrm{EMA}_{\mathrm{n}}$ das farinhas de origem animal, verifica-se que esses valores foram praticamente inferiores aos relatados por Rostagno et al. (2000), pela EMBRAPA (1991) e pelo NRC (1994), com exceção da FVA 3. Comparando os valores de EMA aos de EMV dos 
alimentos, verificou-se que os valores de EMV foram em média 5,22\% superiores aos de EMA. Este resultado, no entanto, não é esperado, principalmente quando se utiliza o método tradicional de coleta total, pois, quando o consumo de alimento é elevado, as perdas energéticas metabólicas e endógenas são anuladas, fazendo com que a EMV seja semelhante à EMA (Lima et al., 1989; Farrel et al., 1991). Segundo Parsons et al. (1982), os valores de EMV são sempre elevados, pois são corrigidos pelas perdas endógenas e metabólicas.

Ao fazer a correção pelo balanço de nitrogênio nos valores de EMV, obtiveram-se os valores de $E_{n}$, que diminuíram drasticamente após a correção, observando-se uma interferência do balanço positivo apresentado pelas aves na retenção do nitrogênio.

Conforme representado na Tabela 4 , houve diferença significativa $(\mathrm{P}<0,05)$ entre os coeficientes de metabolizabilidade dos alimentos. As FP apresentaram os piores coeficientes de metabolizabilidade. O menor coeficiente de metabolizabilidade, o menor conteúdo de EE e o maior valor de DGM foram obtidos com a FP 1, contudo, a FP 3 apresentou maior coeficiente de metabolizabilidade entre as FP estudadas e, conseqüentemente, o menor valor de DGM e o maior conteúdo de EE. As FP são resultantes da cocção, sob pressão, de penas limpas e não decompostas obtidas no abate de aves e geralmente são ricas em proteínas estruturais resistentes e insolúveis, denominadas queratina, que podem resultar em baixos valores de metabolizabilidade.

Segundo Brugalli et al. (1999), as diferenças entre os valores de DGM podem proporcionar variações entre os valores energéticos, pois, quanto maior a partícula, menor a superfície de exposição às ações enzimáticas digestivas e menor a taxa de passagem no trato gastrointestinal das aves. Sabe-se que o tempo de passagem do bolo alimentar pelo trato gastrointestinal das aves é relativamente curto, portanto, a redução do tamanho das partículas pode ter contribuído substancialmente para melhor digestão e absorção dos nutrientes. O tamanho das partículas pode promover alterações no consumo (Penz Jr. et al., 1999) e afetar a utilização da EB (Brugalli et al., 1999).

De acordo com Brugalli et al. (1999), as diferenças entre as FVA e as FVS podem ser explicadas pelos menores valores de DGM das FVA e, conseqüentemente, pelos maiores coeficientes de metabolizabilidade, o que não ocorreu com as FVS, que apresentaram os maiores diâmetros médios e, conseqüentemente, valores de coeficientes de metabolizabilidade inferiores aos das FVA. Nas cinco farinhas de vísceras estudadas, os valores de gordura variaram de 13,26 a $18,44 \%$ e o teor de cinzas nas FVS foi em média $46,36 \%$ maior.

As grandes variações encontradas nos coeficientes de metabolizabilidade dos alimentos estudados podem ainda ser atribuídas aos diferentes processamentos a que os alimentos foram submetidos, resultando em matériasprimas de diferentes qualidades. No processamento das farinhas de origem animal, a temperatura necessária para eliminação dos agentes patogênicos presentes nos resíduos e para promover a quebra das ligações entre os aminoácidos que constituem a proteína das penas, no caso queratinas, geralmente é elevada. Essa alta temperatura proporciona reações entre os nutrientes, formando complexos ou provocando a desnaturação protéica, tornando esses nutrientes indigeríveis e ocasionando redução no valor energético dos alimentos, traduzida

Tabela 4 - Coeficientes de metabolizabilidade da energia metabolizável aparente (CMA), aparente corrigida $\left(C M A_{n}\right)$, verdadeira $(C M V)$ e verdadeira corrigida $\left(\mathrm{CMV}_{\mathrm{n}}\right)$ dos alimentos

Table 4 - Coefficients of metabolizability of apparent metabolizable energy (AMC), corrected apparent energy (AMC ${ }_{n}$ ), true metabolizability energy (TMC) and corrected true energy $\left(T M C_{n}\right)$ of feedstuffs

\begin{tabular}{|c|c|c|c|c|}
\hline $\begin{array}{l}\text { Alimento } \\
\text { Feedstuff }\end{array}$ & $\begin{array}{l}\text { CMA } \\
A M C\end{array}$ & $\begin{array}{l}\mathrm{CMA}_{\mathrm{n}} \\
A M C_{n}\end{array}$ & $\begin{array}{l}\text { CMV } \\
T M C\end{array}$ & $\begin{array}{l}\mathrm{CMV}_{\mathrm{n}} \\
T M C_{n}\end{array}$ \\
\hline Farinha carne e ossos 1 (meal and bone meat 1) & $42,18 \mathrm{~cd}$ & $35,79 \mathrm{de}$ & $45,33 \mathrm{~cd}$ & $36,42 d$ \\
\hline Farinha carne e ossos 2 (meal and bone meat 2) & $60,00 \mathrm{ab}$ & $52,51 \mathrm{bc}$ & $63,40 \mathrm{ab}$ & $54,39 \mathrm{abc}$ \\
\hline Farinha de penas 1 (feather meal 1 ) & $34,70 \mathrm{~d}$ & $30,79 \mathrm{e}$ & $36,82 \mathrm{~d}$ & $18,54 \mathrm{e}$ \\
\hline Farinha de penas 2 (feather meal 2) & $43,41 \mathrm{~cd}$ & $38,19 \mathrm{de}$ & $45,24 \mathrm{~cd}$ & $29,39 \mathrm{de}$ \\
\hline Farinha de penas 3 (feather meal 3) & $48,41 \mathrm{bcd}$ & $41,72 \mathrm{cde}$ & $51,85 \mathrm{bc}$ & $37,78 \mathrm{~d}$ \\
\hline Farinha vísceras aves 1 (poultry by-product 1) & $64,47 \mathrm{a}$ & $60,72 \mathrm{ab}$ & $67,68 \mathrm{a}$ & $56,40 \mathrm{a}$ \\
\hline Farinha vísceras aves 2 (poultry by-product 2) & $63,37 \mathrm{a}$ & $61,01 \mathrm{ab}$ & $66,68 \mathrm{a}$ & $55,06 \mathrm{ab}$ \\
\hline Farinha vísceras aves 3 (poultry by-product 3) & $71,34 \mathrm{a}$ & $63,50 \mathrm{a}$ & $74,58 \mathrm{a}$ & $66,61 \mathrm{a}$ \\
\hline Farinha vísceras suínas 1 (swine víscera meal 1) & $50,34 \mathrm{bc}$ & $48,35 \mathrm{~cd}$ & $53,30 \mathrm{bc}$ & $41,82 \mathrm{bcd}$ \\
\hline Farinha vísceras suínas 2 (swine víscera meal 2) & $49,19 \mathrm{bcd}$ & $45,41 \mathrm{~cd}$ & $52,22 \mathrm{bc}$ & $40,48 d$ \\
\hline Farinha vísceras e penas (feather and víscera meal) & $45,73 \mathrm{~cd}$ & $40,47 \mathrm{de}$ & $47,90 \mathrm{~cd}$ & $35,60 \mathrm{~d}$ \\
\hline CV $(\%)$ & 16,74 & 16,42 & 15,99 & 23,27 \\
\hline
\end{tabular}

Médias seguidas pela mesma letra na coluna não diferem entre si pelo teste de SNK a $5 \%$. Means followed by different letters in the column do not differ $(P<0.05)$ by SNK test. 
pelos coeficientes de metabolizabilidade encontrados (Butolo, 2002).

\section{Conclusões}

Os valores de EMA e EMA ${ }_{\mathrm{n}}$, expressos em $\mathrm{kcal} / \mathrm{kg}$ de MS, foram em média de 1.647 e 1.422 para a farinha de carne e ossos; de 2.143 e 1.875 para a farinha de penas; de 3.062 e 2.844 para a farinha de vísceras de aves; de 1.889 e 1.779 para a farinha de vísceras suínas; e de 2.497 a 2.209 para a farinha de penas e vísceras. Os valores de EMV e EMV expressos em $\mathrm{kcal} / \mathrm{kg}$ de MS, foram em média de 1.753 e 1.462 para a farinha de carne e ossos; de 2.269 e 1.452 para a farinha de penas; de 3.211 e 2.740 para a farinha de vísceras de aves; de 2.002 e 1.562 para a farinha de vísceras suínas; e de 2.615 e 1.944 para a farinha de penas e vísceras.

Os coeficientes de metabolizabilidade da energia bruta foram em média de $49,31 \%$.

\section{Literatura Citada}

ASSOCIAÇÃO NACIONAL DOS FABRICANTES DE RAÇÕES ANFAR. Matérias-primas para alimentação animal padrão. 4.ed. Brasília: Ministério da Agricultura e do Abastecimento, 1985. 65p

BRUGALLI, I.; ALBINO, L.F.T.; SILVA, D.J. et al. Efeito do tamanho de partícula e do nível de substituição nos valores energéticos da farinha de carne e ossos para pintos de corte. Revista Brasileira de Zootecnia, v.28, n.4, p.753-757, 1999.

BUTOLO, J.E. Qualidade de ingredientes na alimentação animal. 1.ed. Campinas: Colégio Brasileiro de Nutrição Animal, 2002. 430 p.

COELHO, M.G.R. Valores energéticos e de triptofano metabolizável de alimentos para aves, utilizando duas metodologias. Viçosa, MG: Universidade Federal Viçosa, 1983. 77p. Dissertação (Mestrado em Zootecnia) - Universidade Federal de Viçosa, 1983.

EMPRESA BRASILEIRA DE PESQUISA AGROPECUÁRIA EMBRAPA. Centro Nacional de Pesquisa de Suínos e Aves (CNPSA) - EMBRAPA-CNPSA. Tabelas de composição química e valores energéticos de alimentos para suínos e aves. 3.ed. Concórdia: EMBRAPA-CNPSA, 1991. 97p. (Documentos, 19).

FARREL, D.J.; THOMPSON, E.; PREEZ, J.J. The estimation of endogenous excreta and the measurement of metabolizable energy in poultry feedstuffs using four feeding systems, four assay methods and four diets. British Poultry Science, v.32, n.3, p.483-499, 1991 .
LEESON; S.; SUMMERS, J.D. Scott's nutrition of the chicken. 4.ed. Guelph: University Books, 2001. 591p.

LIMA, I.L.; SILVA, D.J.; ROSTAGNO, H.S. et al. Composição química e valores energéticos de alguns alimentos determinados com pintos e galos, utilizando duas metodologias. Revista da Sociedade Brasileira de Zootecnia, v.18, n.6, p.546-556, 1989 .

MATTERSON, L.D.; POTTER, L.M.; STUTZ, M.W. et al. The metabolizable energy of feed ingredients for chickens. Storrs: University of Connecticut, 1965. v.11, p.3-11 (Agricultural Experiment Station Research Report).

NASCIMENTO, A.H.; GOMES, P.C.; ALBINO, L.F.T. et al. Química e valores de energia metabolizável das farinhas de penas e vísceras determinados por diferentes metodologias para aves. Revista Brasileira de Zootecnia, v.31, n.3, p.1409-1417, 2002 (supl.).

NATIONAL RESEARCH COUNCIL - NRC. Nutrient requirements of poultry. 9.ed. Washington, D.C.: National Academy of Sciences, 1994. $155 \mathrm{p}$.

NATIONAL RESEARCH COUNCIL - NRC. Nutrient requirements of swine. 10.ed. Washington, D.C.: National Academy of Sciences, 1998. 189p.

PARSONS, C.M.; POTTER, L.M.; BROWN, J.R. Effects of dietary protein and intestinal microflora and excretion of amino acids in poultry. Poultry Science, v.61, n.3, p.639-645, 1982.

PENZ JR., A.M.; KESSLER, A.M.; BRUGALLI, I. Novos conceitos de energia para aves. In: SIMPÓSIO INTERNACIONAL SOBRE NUTRIÇÃO DE AVES, 1999, Campinas. Anais... Campinas: Fundação Avícola de Tecnologia e Ciência Avícolas, 1999. p.1-24.

ROSTAGNO, H.S.; ALBINO, L.F.T.; DONZELE, J.L. et al. Tabelas brasileiras para aves e suínos. Composição de alimentos e exigências nutricionais. Viçosa, MG: Universidade Federal de Viçosa, 2000. 141p.

SCOTT, M.L.; NESHEIM, M.C.; YOUNG, R.J. Nutrition of the chicken. 3.ed. Ithaca: 1982. 562p.

SILVA, D.J.; QUEIROZ, A.C. Análise de alimentos (métodos químicos e biológicos). 1.reimpressão. Viçosa, MG: Universidade Federal de Viçosa, 2002. 235p.

TEIXEIRA, A.S. Tabelas de composição dos alimentos e exigências nutricionais. Alimentos e alimentação dos animais. Lavras: UFLA/FAEPE, 1998. v.2, p.98.

UNIVERSIDADE FEDERAL DE VICOSA - UFV. Manual de utilização do programa SAEG (Sistema para Análise Estatísticas e Genéticas). Viçosa, MG: Imprensa Universitária, 1999. 59p.

ZANOTTO, D.L.; BELlaVER, C. Método de determinação da granulometria de ingredientes para uso em rações de suínos e aves. Concórdia: Embrapa Suínos e Aves, 1996. p.1-5, (Comunicado Técnico) 